\title{
Endoscopic management of colorectal adenomas
}

\author{
Benjamin Meier ${ }^{\text {, }}$ Karel Caca ${ }^{a}$, Andreas Fischer ${ }^{\text {, }}$, Arthur Schmidt ${ }^{\text {b }}$
}

Klinikum Ludwigsburg, Ludwigsburg; University of Freiburg, Faculty of Medicine, University of Freiburg, Germany

\begin{abstract}
Colorectal adenomas are well known precursors of invasive adenocarcinoma. Colonoscopy is the gold standard for adenoma detection. Colonoscopy is far more than a diagnostic tool, as it allows effective treatment of colorectal adenomas. Endoscopic resection of colorectal adenomas has been shown to reduce the incidence and mortality of colorectal cancer. Difficult resection techniques are available, such as endoscopic mucosal resection, endoscopic submucosal dissection and endoscopic full-thickness resection. This review aims to provide an overview of the different endoscopic resection techniques and their indications, and summarizes the current recommendations in the recently published guideline of the European Society of Gastrointestinal Endoscopy.
\end{abstract}

Keywords Polypectomy, endoscopic mucosal resection, endoscopic submucosal dissection, endoscopic full-thickness resection

Ann Gastroenterol 2017; 30 (6): 1-6

\section{Introduction}

Colorectal adenomas are well known precursors of invasive adenocarcinoma. Colonoscopy is the gold standard for adenoma detection and the adenoma detection rate (ADR) is considered to be a quality indicator for screening colonoscopy. In a large prospective cross-sectional study (collecting results from 2,821,392 screening colonoscopies) the ADR was $19.4 \%$ [1]. Colonoscopy is not only a diagnostic tool, but also allows effective treatment of those neoplasms and endoscopic resection of colorectal adenomas has been shown to reduce the incidence and mortality of colorectal cancer [2-5]. This review aims to provide an overview of the endoscopic management of colorectal adenomas and summarizes the current recommendations in the recently published guideline of the European Society of Gastrointestinal Endoscopy (ESGE) [8].

Departments of aGastroenterology, Klinikum Ludwigsburg (Benjamin Meier, Karel Caca); ${ }^{b}$ Medicine II, Medical Center-University of Freiburg, Faculty of Medicine, University of Freiburg (Andreas Fischer, Arthur Schmidt), Germany

\section{Conflict of Interest: None}

Correspondence to: Arthur Schmidt, Department of Medicine II, University of Freiburg, Hugstetter Strasse 55, 79106 Freiburg, e-mail: arthur.schmidt@uniklinik-freiburg.de

Received 15 July 2017; accepted 17 August 2017; published online 23 September 2017

DOI: https://doi.org/10.20524/aog.2017.0193

\section{Definition and endoscopic classification of colorectal adenomas}

Colorectal adenomas are defined as low-grade or highgrade intraepithelial neoplasia. Histologically, a tubular, villous, tubulovillous or serrated architecture can be distinguished. Endoscopically, flat, sessile or pedunculated lesions can be differentiated. Lesion size, tumor morphology and histological findings correlate with the progression to high-grade intraepithelial neoplasia or invasive carcinoma [6,7]. In consequence, it is recommended to document tumor size and describe the morphology of polyps using the Paris classification system [8]. In the Paris classification, mucosal lesions are characterized as pedunculated (Ip), sessile (Is) and nonpolypoid (slightly elevated [IIa], flat [IIb], depressed [IIc]), or excavated/ ulcerated (III) lesions (Fig. 1). For sessile or flat (Paris Is or II) polyps $>10 \mathrm{~mm}$, which are termed laterally spreading tumors (LST), it is recommended to describe surface morphology as granular (LST-G) or non-granular (LST-NG) (Fig. 2). Advanced imaging techniques, such as narrow-band imaging, Fujinon intelligent chromoendoscopy or i-scan, were found to improve the identification of adenomas versus hyperplastic polyps and help to identify morphological features suggestive of malignancy [9-12]. Several classifications have been proposed to predict histologic findings. The "pit-pattern" classification of Kudo [10] or the narrow-band imaging international colorectal endoscopic (NICE) classification allow the prediction of histological findings (hyperplastic polyp, adenoma, invasive carcinoma) by interpretation of tumor color and interpretation of regular, irregular or 


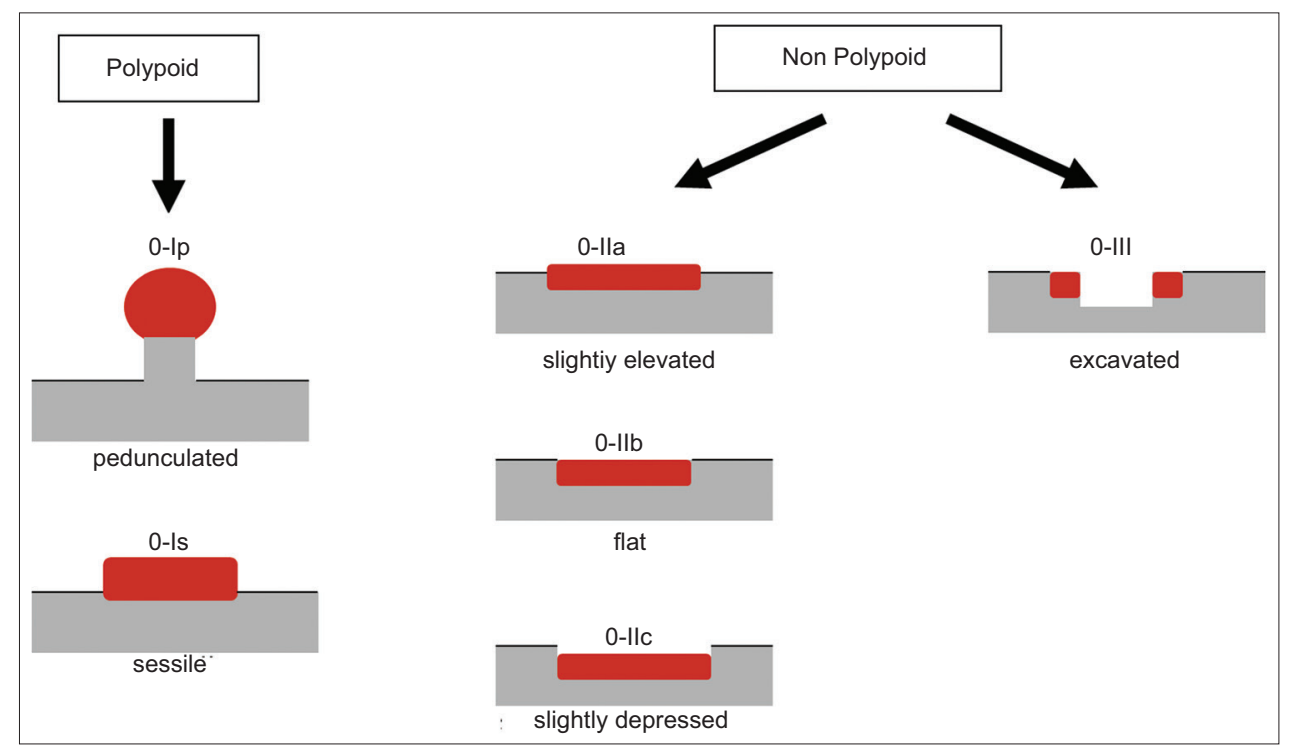

Figure 1 Paris classification

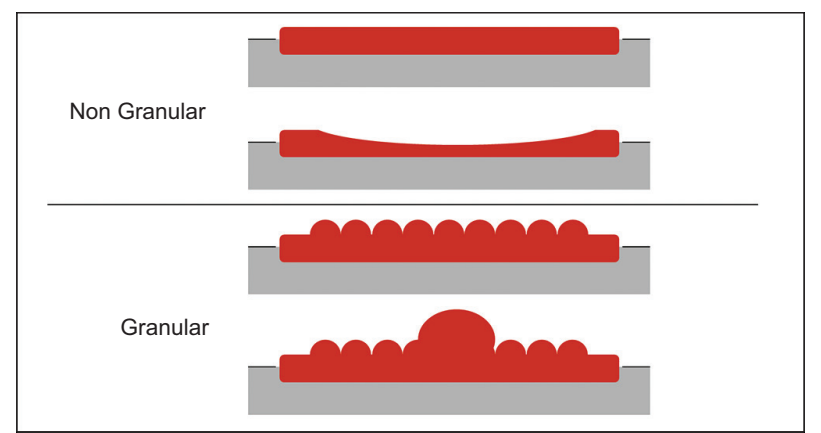

Figure 2 Surface morphology of laterally spreading tumors

absent surface vascular patterns. The WASP (workgroup serrated polyps and polyposis) classification [13] is based on the NICE classification and allows discrimination between serrated and hyperplastic lesions by interpretation of additional characteristics (dark spots inside crypts, irregular shape, indistinct border, clouded surface).

\section{Endoscopic resection}

Different endoscopic resection techniques are available and suggested by international guidelines. The indication for the different techniques is mainly based on the size and morphology of the lesion and on the likelihood of submucosal invasion. The recommendations for endoscopic resection in this article are based mainly on the ESGE guideline [8].

\section{Diminutive polyps}

As diminutive colonic polyps ( $\leq 5 \mathrm{~mm}$ ) exhibit a low risk for cancer, different strategies have been proposed. If they are located in the rectosigmoid, resection is not mandatory when the lesion is predicted to be hyperplastic with high confidence ("diagnose and leave behind"). For all other (diminutive) polyps, endoscopic resection and histopathological examination are recommended. The "resect and discard" strategy may be an alternative strategy for diminutive polyps, but is recommended only for expert centers. The ESGE recommends cold snare polypectomy (CSP) as the preferred technique for polyps $\leq 5 \mathrm{~mm}$, because it has been reported to be superior to cold biopsy forceps $(\mathrm{CBF})$ in terms of complete resection. A randomized controlled trial [14] that included 117 diminutive polyps in 54 consecutive patients showed significantly higher rates of complete resection in the CSP group compared to the $\mathrm{CBF}$ group. The procedure time was also shorter in the CSP group. When CSP is not technically possible or is difficult (polyp size 1-3 mm), the ESGE also suggests $\mathrm{CBF}$ resection. Use of jumbo forceps is then recommended because of their higher complete resection rates compared to standard forceps. For sessile polyps 6-9 $\mathrm{mm}$ in size, snare polypectomy is recommended because $\mathrm{CBF}$ resection is associated with a significantly higher rate of incomplete resection [15]. According to the ESGE guideline, CSP should preferred over hot snare polypectomy (HSP) because of its superior safety profile [16], although clear evidence showing significant superiority of CSP is lacking. The German guideline [17] recommends snare polypectomy and does not comment on CSP vs. HSP. For removal of sessile polyps $10-19 \mathrm{~mm}$ in size, HSP is recommended after submucosal injection to reduce deep thermal injury. However, for polyps with a narrow base, resection without prior injection might be appropriate. Complete "en bloc" resection for polyps $\geq 10 \mathrm{~mm}$ cannot be achieved with CSP. Pedunculated polyps should be resected with HSP. To prevent bleeding, large polyps (head $\geq 20 \mathrm{~mm}$ or stalk $\geq 10 \mathrm{~mm}$ ) should pretreated with injection (saline/epinephrine) or mechanical devices (e.g., plastic loop or clip). 


\section{Endoscopic mucosal resection (EMR) and endoscopic submucosal dissection (ESM)}

Sessile adenomas $\geq 20 \mathrm{~mm}$ or polyps with difficult characteristics (LSTs, non-lifting lesions, difficult location such as ileocecal valve, appendiceal orifice, anorectal junction or behind a fold) should be referred to an experienced endoscopist working in an well-resourced endoscopic center. En bloc resection techniques, such as EMR, ESD or surgery, should always be the techniques of choice in case of suspected superficial invasive carcinoma. The EMR approach (snare resection after submucosal injection) is safe and efficient (Fig. 3). Moreover, it is technically easier to perform compared to ESD. However, for large ( $\geq 20 \mathrm{~mm}$ ) sessile polyps or LST, en bloc EMR is technically not possible-the en bloc resection rate has been reported to be $<32 \%[18,19]$. In those cases piecemeal EMR can be performed, but is associated with higher recurrence rates compared to en bloc resection. A large prospective multicenter Australian study (ACE) included more than 1000 wide-field EMRs (up to $120 \mathrm{~mm}$ in size) and showed a recurrence rate of $16 \%$. However, patients underwent close endoscopic follow up and residual/ recurrent lesions were diminutive and easy to resect in most cases [20]. In the long term, over $90 \%$ of large polyps could be treated endoscopically in this study, supporting the value of piecemeal EMR even for large adenomas.

ESD allows en bloc resection of large ( $\geq 20 \mathrm{~mm}$ ) sessile polyps or LSTs (Fig. 4). Multiple studies have shown lower recurrence rates compared to piecemeal resection. However, the technique is complex, is associated with a significantly longer procedure time and harbors a significantly higher risk of perforation [18]. A Japanese study showed a recurrence rate of $2 \%$ (compared to $14 \%$ after piecemeal EMR) after en bloc ESD of polyps $\geq 20 \mathrm{~mm}$ [21]. The perforation rate was $6.2 \%$ compared to $1.3 \%$ in the EMR group in the same study. Most ESD studies originate from Japan and Korea, where ESD is performed by highly specialized endoscopists. When ESD is performed in the Western world, higher rates of adverse events and lower rates of complete resection are reported [22,23]. The meta-analysis of Fuccio et al found a significantly lower rate of complete resection (71.3\% vs. $85.6 \%)$ and a significantly higher need for surgical treatment after polypectomy (3.1\% vs. $0.8 \%$ ) in non-Asian vs. Asian countries. It is important to note that ESD in the rectum is much easier to perform compared with colonic ESD [24]. In the rectum, the wall is thicker, endoscope maneuverability is better and small perforations are generally clinically not relevant. Colonic ESD is associated with a higher frequency of complications and lower complete resection rates. A large German case series reported by Sauer et al (182 patients, mainly colonic lesions) showed perforation in $9.3 \%$ for lesions $\geq 20 \mathrm{~mm}$ and complete resection in only $62.6 \%$. With the progressing learning curve in the Western world, positive developments of these endpoints have also been reported [23,25-27].

\section{T1 carcinomas}

In cases of suspected superficial carcinoma, the lesion should be resected en bloc; piecemeal EMR cannot be

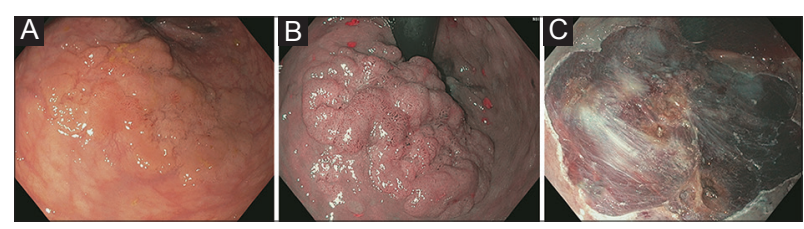

Figure 3 Endoscopic mucosal resection of a laterally spreading tumor (LST) in the rectum. LST in white light (A) and narrow band imaging (B). Macroscopic complete resection after piecemeal endoscopic mucosal dissection (C). Histology: tubulovillous adenoma with low-grade dysplasia

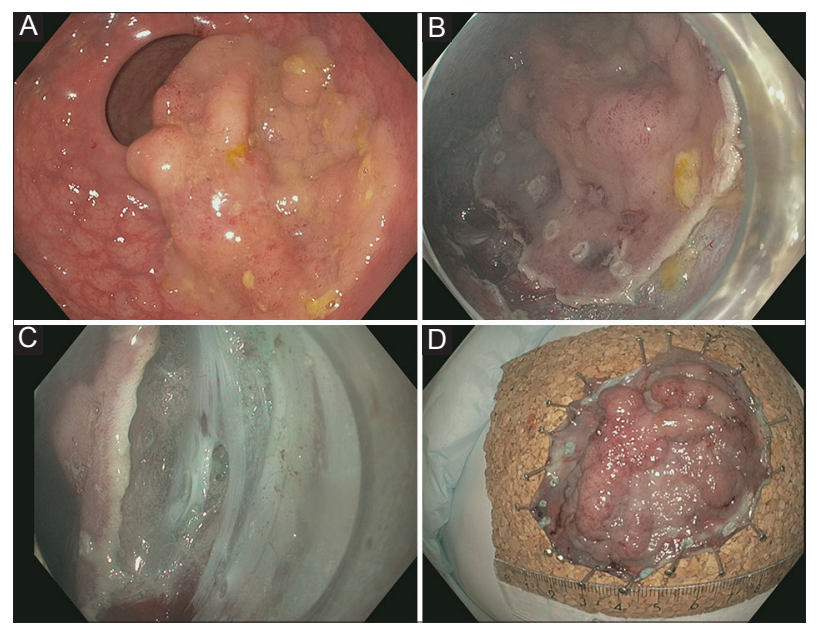

Figure 4 Endoscopic submucosal dissection (ESD) of a laterally spreading tumor (LST) in the rectum. LST in white light, tumor size about $50 \mathrm{~mm}$ (A). The lesion is marked circumferentially by coagulation. After injection, the mucosa is cut circumferentially with an electrosurgical knife (B). The electrosurgical knife is then used for submucosal dissection (C). Macroscopic complete resection after ESD (D). Histology: Complete resection of a T1-carcinoma with low-risk criteria (G2 T1 L0 V0 R0, submucosal invasion: $800 \mu \mathrm{m}$ )

recommended in this situation. For lesions $<2 \mathrm{~cm}$ en bloc EMR may be used; alternatively, clip-assisted full-thickness resection can be performed (see below). ESD allows en bloc resection of larger lesions but should be performed by endoscopists with sufficient expertise, especially when the lesion is located proximally to the rectum. Alternatively, surgical resection can be performed; data showing better outcomes for ESD are lacking. The final histological report of the resection specimen should contain information about tumor infiltration depth (T-stage and depth of submucosal invasion in $\mu \mathrm{m}$, grading and evidence of lymphovascular invasion). Patients with incomplete resection or those at high risk of lymph-node metastasis (submucosal invasion $>1000 \mu \mathrm{m}$ or lymphovascular invasion or undifferentiated [G3] tumors) should be referred for oncological surgical resection. Lesions should be tattooed before surgical resection, except when located in the cecum or the rectum. After the formation of a submucosal bleb with saline (to avoid complications such as peritonitis), the injection of sterile carbon particle suspension is recommended. The lesions should be tattooed $3 \mathrm{~cm}$ distally with 2 or 3 injections [8]. In patients with suspected deep 
submucosal infiltration, endoscopic resection should not be attempted.

\section{Endoscopic full-thickness resection (EFTR)}

EFTR allows the treatment of colorectal lesions with a size $<30 \mathrm{~mm}$ that are not resectable using the standard procedures. These lesions are typically "non-lifting" lesions, because of submucosal scarring (usually pretreated and recurrent lesions) or submucosal tumor invasion. Other indications for EFTR are lesions in a difficult location (diverticulum or appendiceal orifice) or subepithelial tumors. The Full-Thickness Resection Device (FTRD ${ }^{\circledR}$, Ovesco Endoscopy AG, Tübingen, Germany) is the only device available for resection in the colorectum. It is an over-the-scope system that allows single-step EFTR after placement of a modified over-the-scope clip (OTSC ${ }^{\circledast}$ ) (Fig. 5). The device has been described to be feasible and effective in a case series and two small retrospective clinical studies [28-31]. A prospective, non-randomized German multicenter ( 9 centers) study (WALL RESECT, NCT02362126) included 181 patients who underwent EFTR (104 nonlifting lesions, 39 with difficult location, 23 subepithelial tumors, 15 superficial carcinomas). The lesions were located throughout the colorectum and the mean lesion size was $15 \mathrm{~mm}$ (range 2-30 mm). All lesions could be reached and resected macroscopically complete in almost $90 \%$ using the FTRD. The histological complete resection rate was $76.9 \%$ overall. For lesions $<20 \mathrm{~mm}$ histological complete resection could be achieved in almost $85 \%$ ( $100 \%$ for lesions $<5 \mathrm{~mm})$. Postprocedural bleeding was reported in 4 cases $(2.2 \%)$ and was managed endoscopically. Perforation occurred in 6 cases (3.3\%) and required surgery in 2 cases. After resection close to the appendix (34 cases), appendicitis occurred in 3 cases $(8.8 \%)$ and surgical resection was required in 1 case. After 3 months, almost $70 \%$ of the clips had detached spontaneously. Residual lesions were observed in $12.5 \%$; all but 3 patients could be retreated endoscopically. The authors concluded that, for lesions not amenable to conventional endoscopic resection, EFTR with the FTRD shows good technical efficacy (especially in lesions $<20 \mathrm{~mm}$ ) with acceptable complication rates [32].

\section{Management of residual or recurrent lesions}

Piecemeal resection of large adenomas is associated with moderate rates of recurrence (about 16\%) [20]. Recurrent or residual lesions are usually diminutive in size and can be easily resected in most cases during surveillance endoscopy using the standard techniques. However, recurrent adenomas can show a "non-lifting" sign because of submucosal scarring. In this situation, EFTR is a powerful option for en bloc resection and provides a good alternative to ESD or surgical resection. However, comparative studies are lacking. Different supplementary ablative strategies after piecemeal resection have been described. Argon plasma coagulation and avulsion

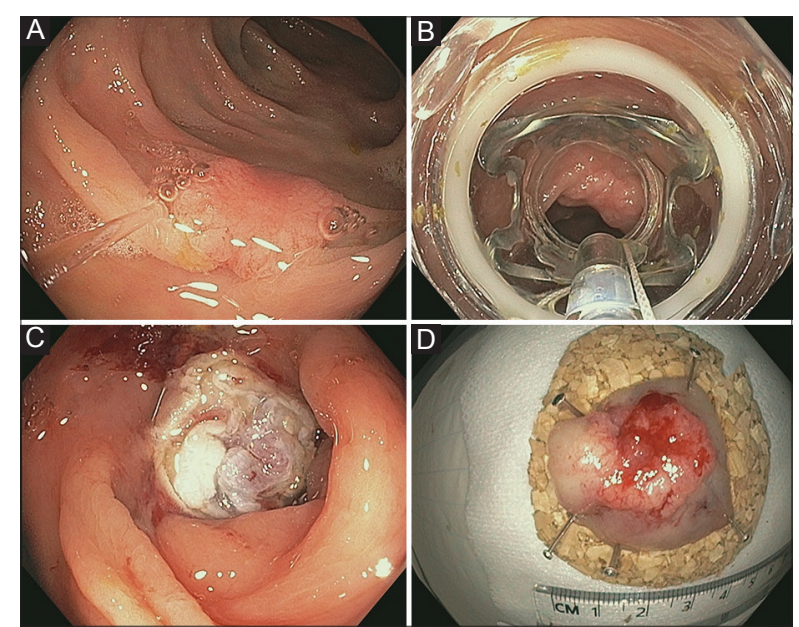

Figure 5 Full-thickness resection of a non-lifting adenoma in the transverse colon with the Full-Thickness Resection Device (FTRD). Lesion in white light (Paris 0 -IIa/IIc), size about $20 \mathrm{~mm}$ (A). The lesion is identified with the mounted FTRD (B) and then grasped with forceps. The lesion is then pulled completely into the cap. A duplication of the gastrointestinal wall is created. The over-the-scope clip (OTSC) is deployed and the lesion is resected above the OTSC. Resection site after resection with the FTRD (C). Macroscopic complete resection after endoscopic full-thickness resection (D). Histology: Complete resection of a T1-carcinoma with low-risk criteria (G2 T1 L0 V0 R0, submucosal invasion: $700 \mu \mathrm{m}$ )

(using electrocautery biopsy forceps) are the most common techniques. They can be used for tissue not amenable to snare resection ("adjunct treatment") or for cleaning defect margins ("adjuvant treatment"). However, when using adjunct argon plasma coagulation, recurrence rates up to $50 \%$ are reported $[33,34]$. Small studies have shown that hot avulsion may be more effective for small residual "non-lifting" adenomas after piecemeal EMR [35-37].

\section{Polypectomy-associated adverse events}

Intraprocedural bleeding occurs in $2.8 \%$ when standard polypectomy is performed [38] and may increase to $11.3 \%$ for lesions $\geq 20 \mathrm{~mm}$ when EMR is performed [39]. However, bleeding is rarely serious and can effectively be treated with endoclips or coagulation. In low-risk situations (standard polypectomy of sessile polyps $\leq 20 \mathrm{~mm}$ ), mechanical clip closure for prophylaxis is usually not necessary. In high-risk situations (such as tumor size $\geq 20 \mathrm{~mm}$, comorbidities, proximal location, anticoagulation or dual antiplatelet therapy) mechanical clip closure should be considered individually. After resection the resection site should be carefully inspected for risk factors of imminent perforation. When risk factors are identified (such as exposure of the muscularis propria, submucosal fibrosis, submucosal fat, or attempted en bloc snare resection of lesions $\geq 25 \mathrm{~mm}$ ), prophylactic clip closure should be performed. According to a large meta-analysis, perforation after EMR can be expected in $1.4 \%$ and in $5.7 \%$ after ESD [40]. If it is 
diagnosed immediately (or within $4 \mathrm{~h}$ ), endoscopic clip closure can be performed. For smaller perforations $(<10 \mathrm{~mm})$ throughthe-scope-clips are adequate, for perforations $>10 \mathrm{~mm}$ an OTSC should be used [41]. In addition, intravenous fluids and broad spectrum antibiotics are administered and the patient is monitored. Clear indications for surgery are complications or a failed endoscopic closure.

\section{Surveillance after polypectomy}

Recommended surveillance intervals are determined by adenoma size, histological findings, number of resected adenomas and completeness of resection. The recommendations for surveillance after polypectomy in this article are based on the ESGE and German guidelines [8,17]. After resection of hyperplastic polyps $<10 \mathrm{~mm}$, surveillance after 10 years is adequate if there is no family history of colorectal cancer (German guideline). After en bloc resection of a maximum of 2 adenomas $<10 \mathrm{~mm}$ without high-grade dysplasia, surveillance after 5 years (German guideline) or 10 years (ESGE guideline) is recommended. For adenomas with serrated histology, endoscopy after 3 years is recommended (German guideline). After en bloc resection of 3-10 adenomas (or at least one $>10 \mathrm{~mm}$ ), or resection of an adenoma with a villous/tubulovillous architecture or highgrade dysplasia, surveillance after 3 years is recommended (ESGE and German guidelines). After incomplete (histologically or macroscopically) or piecemeal resection, surveillance must be performed after 2-6 months (ESGE and German guidelines). If the first surveillance endoscopy shows no recurrence and no residual lesion, endoscopy can be performed every 5 years thereafter. If the first surveillance endoscopy shows a "high-risk" adenoma (villous histology, high-grade dysplasia, $\geq 10 \mathrm{~mm}, \geq 3$ adenomas), this interval should be reduced to 3 years (ESGE guideline). After resection of $>10$ adenomas, surveillance must be performed within 3 years (German guideline). Family history and risk factors for colorectal cancer (such as familial adenomatous polyposis, Lynch syndrome, hereditary nonpolyposis colorectal cancer, or inflammatory bowel disease) should be examined and further surveillance should be performed according to the corresponding guidelines. After resection of $>10$ adenomas patients should be referred for genetic counseling, according to the ESGE guideline. After complete resection of a superficial carcinoma with lowrisk features (submucosal invasion $<1000 \mu \mathrm{m}, \mathrm{G} 1$ or G2, no lymphovascular invasion), surveillance after 6 months is necessary (then every 2 years) according to the German guideline.

\section{References}

1. Pox CP, Altenhofen L, Brenner H, Theilmeier A, Von Stillfried D, Schmiegel W. Efficacy of a nationwide screening colonoscopy program for colorectal cancer. Gastroenterology 2012;142:14601467.e2.

2. Atkin WS, Morson BC, Cuzick J. Long-term risk of colorectal cancer after excision of rectosigmoid adenomas. $N$ Engl $J$ Med 1992;326:658-662

3. Citarda F, Tomaselli G, Capocaccia R, Barcherini S, Crespi M; Italian Multicentre Study Group. Efficacy in standard clinical practice of colonoscopic polypectomy in reducing colorectal cancer incidence. Gut 2001;48:812-815.

4. Cottet V, Jooste V, Fournel I, Bouvier AM, Faivre J, Bonithon-Kopp C. Long-term risk of colorectal cancer after adenoma removal: a population-based cohort study. Gut 2012;61:1180-1186.

5. Løberg M, Kalager M, Holme, Hoff G, Adami HO, Bretthauer M. Long-term colorectal-cancer mortality after adenoma removal. N Engl J Med 2014;371:799-807.

6. Jørgensen OD, Kronborg O, Fenger C. The Funen adenoma follow-up study. Characteristics of patients and initial adenomas in relation to severe dysplasia. Scand J Gastroenterol 1993;28:239-243.

7. O'Brien MJ, Winawer SJ, Zauber AG, et al. The National Polyp Study. Patient and polyp characteristics associated with highgrade dysplasia in colorectal adenomas. Gastroenterology 1990;98:371-379.

8. Ferlitsch M, Moss A, Hassan C, et al. Colorectal polypectomy and endoscopic mucosal resection (EMR): European Society of Gastrointestinal Endoscopy (ESGE) Clinical Guideline. Endoscopy 2017;49:270-297.

9. Kudo S, Hirota S, Nakajima T, et al. Colorectal tumours and pit pattern. J Clin Pathol 1994;47:880-885.

10. Kudo S, Tamura S, Nakajima T, Yamano H, Kusaka H, Watanabe H. Diagnosis of colorectal tumorous lesions by magnifying endoscopy. Gastrointest Endosc 1996;44:8-14.

11. Kudo S, Lambert R, Allen JI, et al. Nonpolypoid neoplastic lesions of the colorectal mucosa. Gastrointest Endosc 2008;68:S3-S47.

12. Li M, Ali SM, Umm-a-OmarahGilani S, Liu J, Li YQ, Zuo XL. Kudo's pit pattern classification for colorectal neoplasms: a metaanalysis. World J Gastroenterol 2014;20:12649-12656.

13. IJspeert JE, Bastiaansen BA, van Leerdam ME, et al; Dutch Workgroup serrAted polypS \& Polyposis (WASP). Development and validation of the WASP classification system for optical diagnosis of adenomas, hyperplastic polyps and sessile serrated adenomas/polyps. Gut 2016;65:963-970.

14. Lee CK, Shim JJ, Jang JY. Cold snare polypectomy vs. Cold forceps polypectomy using double-biopsy technique for removal of diminutive colorectal polyps: a prospective randomized study. Am J Gastroenterol 2013;108:1593-1600.

15. Kim JS, Lee BI, Choi H, et al. Cold snare polypectomy versus cold forceps polypectomy for diminutive and small colorectal polyps: a randomized controlled trial. Gastrointest Endosc 2015;81:741-747.

16. Ichise Y, Horiuchi A, Nakayama Y, Tanaka N. Prospective randomized comparison of cold snare polypectomy and conventional polypectomy for small colorectal polyps. Digestion 2011;84:78-81.

17. Leitlinienprogramm Onkologie (Deutsche Krebsgesellschaft, Deutsche Krebshilfe, AWMF): S3-Leitlinie Kolorektales Karzinom, Kurzversion 1.1, 2014, AWMF Registrierungsnummer: 021007OL, http://leitlinienprogrammonkologie.de/Leitlinien.7.0.html

18. Holmes I, Friedland S. Endoscopic mucosal resection versus endoscopic submucosal dissection for large polyps: a western colonoscopist's view. Clin Endosc 2016;49:454-456.

19. Zhan T, Hielscher T, Hahn F, et al. Risk Factors for Local Recurrence of Large, Flat Colorectal Polyps after Endoscopic Mucosal Resection. Digestion 2016;93:311-317.

20. Moss A, Williams SJ, Hourigan LF, et al. Long-term adenoma recurrence following wide-field endoscopic mucosal resection 
(WF-EMR) for advanced colonic mucosal neoplasia is infrequent: results and risk factors in 1000 cases from the Australian Colonic EMR (ACE) study. Gut 2015;64:57-65.

21. Saito Y, Fukuzawa M, Matsuda T, et al. Clinical outcome of endoscopic submucosal dissection versus endoscopic mucosal resection of large colorectal tumors as determined by curative resection. Surg Endosc 2010;24:343-352.

22. Fuccio L, Hassan C, Ponchon T, et al. Clinical outcomes after endoscopic submucosal dissection for colorectal neoplasia: a systematic review and meta-analysis. Gastrointest Endosc 2017;86:74-86..

23. Sauer M, Hildenbrand R, Oyama T, Sido B, Yahagi N, Dumoulin FL. Endoscopic submucosal dissection for flat or sessile colorectal neoplasia $>20 \mathrm{~mm}$ : A European single-center series of 182 cases. Endosc Int Open 2016;4:E895-E900.

24. Pimentel-Nunes P, Dinis-Ribeiro M, Ponchon T, et al. Endoscopic submucosal dissection: European Society of Gastrointestinal Endoscopy (ESGE) Guideline. Endoscopy 2015;47:829-854.

25. Barret M, Lepilliez V, Coumaros D, et al; Société Française $\mathrm{d}^{\prime}$ Endoscopie Digestive (SFED). The expansion of endoscopic submucosal dissection in France: A prospective nationwide survey. United European Gastroenterol J 2017;5:45-53.

26. Probst A, Golger D, Anthuber M, Märkl B, Messmann H. Endoscopic submucosal dissection in large sessile lesions of the rectosigmoid: learning curve in a European center. Endoscopy 2012;44:660-667.

27. Probst A, Ebigbo A, Märkl B, et al. Endoscopic submucosal dissection for early rectal neoplasia: experience from a European center. Endoscopy 2017;49:222-232.

28. Richter-Schrag HJ, Walker C, Thimme R, Fischer A. [Full thickness resection device (FTRD). Experience and outcome for benign neoplasms of the rectum and colon]. Chirurg 2016;87:316-325.

29. Schmidt A, Damm M, Caca K. Endoscopic full-thickness resection using a novel over-the-scope device. Gastroenterology 2014;147:740-742.

30. Schmidt A, Riecken B, Damm M, Cahyadi O, Bauder M, Caca K. Endoscopic removal of over-the-scope clips using a novel cutting device: a retrospective case series. Endoscopy 2014;46:762-766.

31. Schmidt A, Bauerfeind P, Gubler C, Damm M, Bauder M, Caca K. Endoscopic full-thickness resection in the colorectum with a novel over-the-scope device: first experience. Endoscopy 2015;47:719-725.

32. Schmidt A. Colonoscopic full-thickness resection using an overthe-scope device: A prospective multicenter study in various indications. Gut 2017 Aug 10. [Epub ahead of print] doi: 10.1136/ gutjnl-2016-313677

33. Buchner AM, Guarner-Argente C, Ginsberg GG. Outcomes of EMR of defiant colorectal lesions directed to an endoscopy referral center. Gastrointest Endosc 2012;76:255-263.

34. Zlatanic J, Waye JD, Kim PS, Baiocco PJ, Gleim GW. Large sessile colonic adenomas: use of argon plasma coagulator to supplement piecemeal snare polypectomy. Gastrointest Endosc 1999;49:731-735.

35. Andrawes S, Haber G. Avulsion: a novel technique to achieve complete resection of difficult colon polyps. Gastrointest Endosc 2014;80:167-168.

36. Tsiamoulos ZP, Rameshshanker R, Gupta S, Saunders BP. Augmented endoscopic resection for fibrotic or recurrent colonic polyps using an ablation and cold avulsion technique. Endoscopy 2016;48 Suppl 1:E248-E249.

37. Veerappan SG, Ormonde D, Yusoff IF, Raftopoulos SC. Hot avulsion: a modification of an existing technique for management of nonlifting areas of a polyp (with video). Gastrointest Endosc 2014;80:884-888.

38. Di Giorgio P, De Luca L, Calcagno G, Rivellini G, Mandato M, De Luca B. Detachable snare versus epinephrine injection in the prevention of postpolypectomy bleeding: a randomized and controlled study. Endoscopy 2004;36:860-863.

39. Burgess NG, Metz AJ, Williams SJ, et al. Risk factors for intraprocedural and clinically significant delayed bleeding after wide-field endoscopic mucosal resection of large colonic lesions. Clin Gastroenterol Hepatol 2014;12:651-661.e1-e3.

40. Fujiya M, Tanaka K, Dokoshi T, et al. Efficacy and adverse events of EMR and endoscopic submucosal dissection for the treatment of colon neoplasms: a meta-analysis of studies comparing EMR and endoscopic submucosal dissection. Gastrointest Endosc 2015;81:583-595.

41. Paspatis GA, Dumonceau JM, Barthet M, et al. Diagnosis and management of iatrogenic endoscopic perforations: European Society of Gastrointestinal Endoscopy (ESGE) Position Statement. Endoscopy 2014;46:693-711. 